\title{
Automated Early Detection of Diabetic Retinopathy Using Image Analysis Techniques
}

\author{
Neera Singh \\ Indian Institute of Information \\ Technology \\ Allahabad
}

\author{
Ramesh Chandra Tripathi \\ Indian Institute of Information \\ Technology \\ Allahabad
}

\begin{abstract}
Diabetic retinopathy (DR) is a common retinal complication associated with diabetes. It is a major cause of blindness in middle as well as older age groups. Therefore early detection through regular screening and timely intervention will be highly beneficial in effectively controlling the progress of the disease. Since the ratio of people afflicted with the disease to the number of eye specialist who can screen these patients is very high, there is a need of automated diagnostic system for diabetic retinopathy changes in the eye so that only diseased persons can be referred to the specialist for further intervention and treatment.

Various aspects and stages of retinopathy are analyzed by examining the colored retinal images. Microaneurysms are small saccular pouches caused by local distension of capillary walls and appear as small red dots. Their walls are thin and rupture easily to cause hemorrhages. Hard exudates are yellow lipid deposits which appear as bright yellow lesions. The bright circular region from where the blood vessels emanate is called the optic disk. The fovea defines the center of the retina, and is the region of highest visual acuity. The spatial distribution of exudates and microaneurysms and hemorrhages, especially in relation to the fovea can be used to determine the severity of diabetic retinopathy.

Image analysis tools can be used for automated detection of these various features and stages of Diabetes Retinopathy and can be referred to the specialist accordingly for intervention, thus making it a very effective tool for effective screening of Diabetic Retinopathy patients. DR patients require frequent, at least six monthly screening of vast number of patients and automating the process will go a long way in relieving the burden on the specialist and reducing the most common cause of preventable blindness.
\end{abstract}

\section{General Terms}

Image processing, Automated Diagnostic System

\section{Keywords}

Diabetic Retinopathy (DR); Optic Disc; Exudates; Micro aneurysms; Hemorrhages; Retinal Grading Algorithm (RGA)

\section{INTRODUCTION}

Diabetes is fast becoming an epidemic around the world and especially in the Indian society. This is leading to a plethora of Diabetes related disorders like Diabetic Retinopathy (DR), nephropathy and neuropathy etc. These complications if not dealt with on time can lead to lot of disability on the part of the patient and huge cost and work load on the specialists and the government. Hence there is a need to develop an automated diagnostic system (for DR in this case) to expedite the work of the practitioner and reduce morbidity of the patients.

\section{BACKGROUND}

Diabetic Retinopathy lesions vary from microaneurysm and hemorrhages to exudates-soft cotton wool spots and hard exudates (see Figure1.). An automated diagnostic system is used to extract these features in a diabetic retinal picture. In order to extract the abnormal lesion first the normal physiological components resembling the lesion in pixel intensity have to be extracted e.g. optic disc in case of exudates detection and fovea and blood vessels in case of hemorrhages and micro aneurysms.

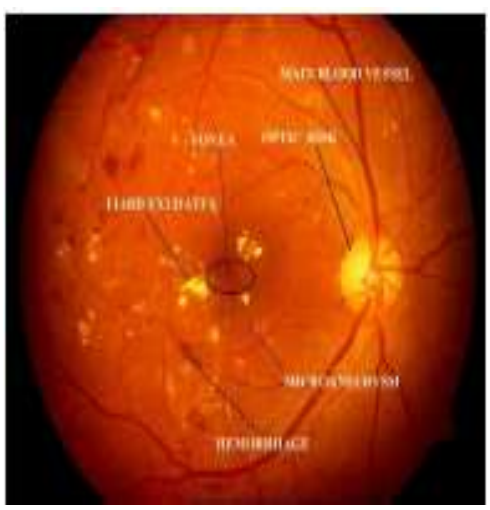

Figure1. Various features of a typical retinopathy image

In [1], the optic disc is localized exploiting its high grey level variation. This approach has been shown to work well, if there are no or only few pathologies like exudates that also appear very bright and are also well contrasted. No method is proposed for the detection of the contours.

In [2], an area threshold is used to localize the optic disc. The contours are detected by means of the Hough transform, i.e., the gradient of the image is calculated, and the best fitting circle is determined. This approach is quite time consuming and it relies on conditions about the shape of the optic disc that are not always met. Sometimes, the optic disc is even not visible entirely in the image plane, and so the shape is far from being circular or even elliptic.

Also, in [3], the Hough transform is used to detect the contours of the optic disc in infrared and argon-blue images. Despite of some improvements, problems have been stated if the optic disc does not meet the shape conditions (e.g., if it lies on the border of the image) or if contrast is very low. 
In [4], the optic disc is localized by back tracing the vessels to their origin. This is certainly one of the safest ways to localize the optic disc, but it has to rely on vessel detection. It is desirable to separate segmentation tasks in order to avoid an accumulation of segmentation errors and to save computational time (the detection of the vascular tree is particularly time consuming).

In [5], morphological filtering techniques and active contours are used to find the boundary of the optic disc, in [19] an area threshold is used to localize the optic disc and the watershed transformation to find its contours. The following are the mathematical morphological operators:-

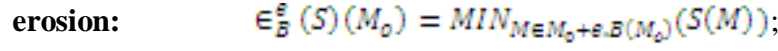

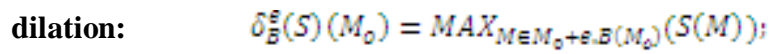

$$
\begin{aligned}
& \text { opening: } \quad v_{B}^{e}(S)=\Phi_{E}^{E}\left(\epsilon_{B}^{Q}(S)\right) \text {; } \\
& \text { closing: } \quad \varphi_{B}^{D}(S)=E_{B}^{D}\left(D_{B}^{D}(S)\right) \text {; } \\
& \text { top-hat: } \quad T H_{B}^{Q}(S)=s-\gamma_{B}^{G}(S) \text {; }
\end{aligned}
$$

In [6], the method produces segmentations by classifying each image pixel as vessel or nonvessel, based on the pixel's feature vector. Feature vectors are composed of the pixel's intensity and two-dimensional Gabor wavelet transform responses taken at multiple scales. A Bayesian classifier with class-conditional probability density functions (likelihoods) described as Gaussian mixtures, yields a fast classification, while being able to model complex decision surfaces.

In order to define vessel-like patterns as in [7], segmentation will be performed with respect to a precise model. A vessel is defined as a bright pattern, piece-wise connected, and locally linear. In order to differentiate vessels from analogous background patterns, a cross-curvature evaluation is performed. They are separated out as they have a specific Gaussian-like profile whose curvature varies smoothly along the vessel. The detection algorithm has been based on four steps: Noise reduction, linear pattern with Gaussian-like profile improvement, cross-curvature evaluation and linear filtering (see figure 2).

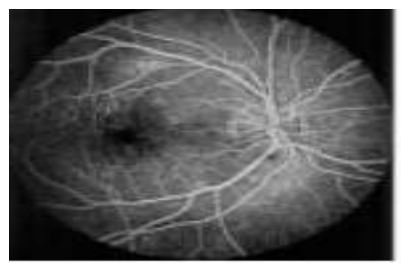

(a)
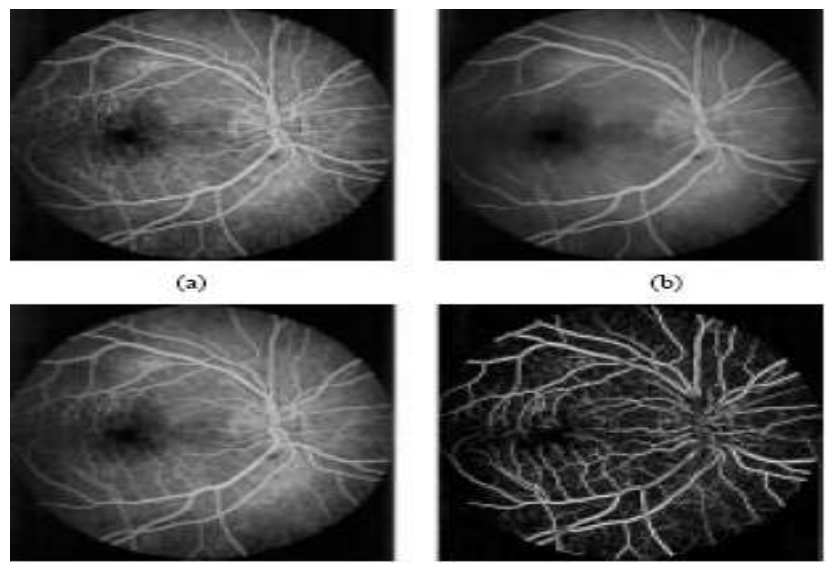

(b)

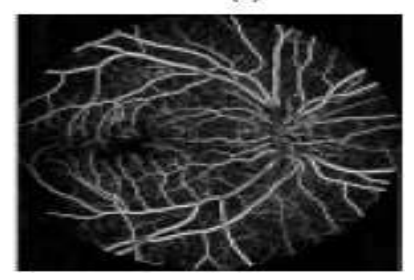

Figure 2. Each step of the morphological treatment: (a) initial image, (b) supremum of opening, (c) reconstruction, and (d) sum of top-hats ${ }^{7}$

In [8],an operator for feature extraction based on the optical and spatial properties of objects to be recognized. The gray-level profile of the cross section of a blood vessel is approximated by a

Gaussian shaped curve. The concept of matched filter detection of signals is used to detect piecewise linear segments of blood vessels in these images. 12 different templates have been constructed that are used to search for vessel segments along all possible directions (see figure 3 ).
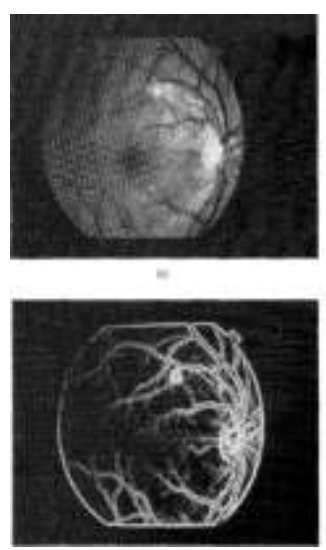

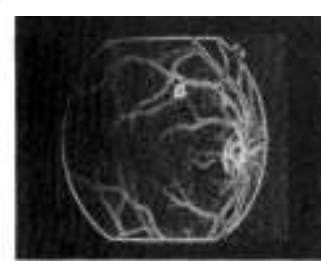

$+n$

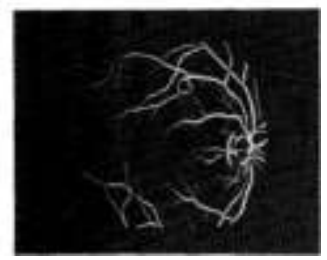

Figure3. Three different filters are applied to a different fundus image: (a) the original image; (b) edge detection by using Sobel operator; (c) edge detection by using morphological edge detector; and (d) detection of blood vessels by using the proposed 2-D matched filter ${ }^{8}$.

In [9], the specific sequence of morphological filters enables the detection of lesions that are brighter or darker than their local background. The incorporation of size constraints at the grayscale processing level results in the rejection of significantly greater numbers of false alarms corresponding to irrelevant background as well as the increased detection performance for low contrast lesions. The analysis consists of three key computational elements; lesion cueing, coarse, and fine segmentation.

In [10], for the detection of the optic disc, first the approximate position is found and then the exact contours are determined by means of the watershed transformation. The algorithm for vessel detection consists in contrast enhancement, application of the morphological top-hat-transform and a postfiltering step in order to distinguish the vessels from other blood containing features (see figure 4).

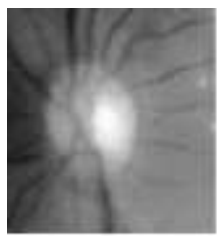

(a)

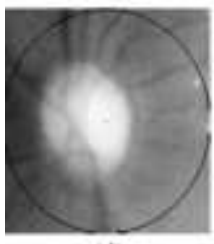

(d)

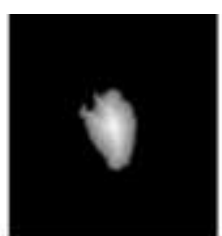

(b)

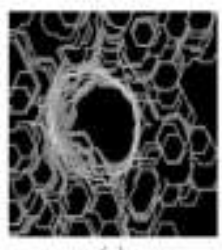

(6)

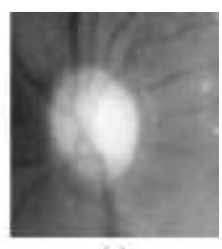

(e)

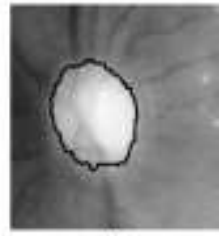

(f)
Figure 4. Figure4.The detection of the optic disc: (a) Luminance channel (b) Distance image of the biggest particle (c) red channel (d) red channel with imposed marker (e) morphological gradient $(f)$ result of segmentation ${ }^{10}$ 
In [11], the use of multiscale amplitude-modulation frequencymodulation (AM-FM) methods for discriminating between normal and pathological retinal images has been proposed. The region types included: microaneurysms, exudates, neovascularization on the retina, hemorrhages, normal retinal background, and normal vessels patterns. The cumulative distribution functions of the instantaneous amplitude, the instantaneous frequency magnitude, and the relative instantaneous frequency angle from multiple scales were used as texture features vectors. Distance metrics between the extracted feature vectors were used to measure interstructure similarity.

\subsection{Detection of hard and soft exudates}

\subsubsection{Optic Disc Localization}

Optic disc shares the same pixel intensity as the hard and soft exudates in retinopathy therefore it becomes mandatory to localize the Optic Disc (which is a normal feature of the retina) for the localization and detection of exudates. Optic Disc can be localized by the following methods:-

1. Hausdorff's Distances-Average Optic Disc diameter is determined from the Optic Disc database and superimposed on the higher pixel intensity areas of the retina.

- Given two sets of points $\mathbf{A}=\left\{\mathbf{a}_{1} \ldots \ldots \mathbf{a}_{\mathbf{m}}\right\}$ and $\mathbf{B}=\left\{\mathbf{b}_{1} \ldots . . \mathbf{b}_{\mathbf{n}}\right\}$, the Hausdorff Distance is defined as $\mathbf{H}(\mathbf{A}, \mathbf{B})=\max (\mathbf{h}(\mathbf{A}, \mathbf{B}), \mathbf{h}(\mathbf{B}, \mathbf{A}))$ Where

$$
h(A, B)=\max _{a \in A} \min _{b \in B}\|a-b\|
$$

- $\|\mathrm{a}-\mathrm{b}\|$ is any metric between the points a and $\mathrm{b}$. For simplicity

$$
\|\mathrm{a}-\mathrm{b}\|=\sqrt{\left(x_{2}-x_{1}\right)^{2}+\left(y_{2}-y_{1}\right)^{2}}
$$

$\|\mathrm{a}-\mathrm{b}\|$ is the Euclidean distance

between $\mathrm{a}(\mathrm{x} 1, \mathrm{y} 1)$ and $\mathrm{b}(\mathrm{x} 2, \mathrm{y} 2)$

For 2 similar point sets that are correctly aligned, $h(A, B)$ becomes small and the minimum distance is thus determined to localize the Optic Disc.

\section{Genetic Algorithm based Location of the Optic Disc}

The pixels with the highest $2 \%$ gray levels in the red component are selected to localize the optic disc. The nearby pixels are assembled into clusters [12]. The clusters with their centroids within a particular distance are grouped to form one cluster. Then the number of pixels in a cluster is ascertained and those with number of pixels less than 100 are discarded. All the remaining clusters are the plausible candidates for the optic disc region [13]. The centroid of the largest cluster is the optic disc center. In order to avoid the possibility of missing the optic disc, clusters with number of pixel more than 100 are also considered.

Genetic Algorithms work with a population of individuals, each representing a possible solution to a given problem. Each individual is assigned a fitness score based on how good the solution is for a particular problem [12]. The fittest individuals are selected for breeding and producing new individuals as offspring and also have some common characteristic with the parent.

\section{[a] Initial Population}

The initial population of the Genetic algorithm is generated based on solutions that lie close to the target, that is, a random set of contours that are close to the sought for contour. As the diameter of optic disc is in the range of 65 100 pixels in the retinal image of 512 x 512 pixels, the initial contours are generated within this limit from the optic disc center[14].

[b] Fitness and Selection

The selection is to consider the fittest (lowest cost) contour, where the cost is associated with the cumulative local intensity differences. The idea of optimal contour is a set of pixels that are both linearly connected and of very similar intensity [12]. The fitness is calculated and those below that level are eliminated and the remaining are taken up for the next iteration till the optimization algorithm converges.

\section{Optic Disc detection and boundary estimation using geometric active contours}

The localization of optic disc by finding the largest cluster of the brightest pixels gives good results with normal retinal images but when the size of the lesion is larger than the optic disc or the optic disc is covered by blood vessels, the method doesn't work well. In that case the method of finding the branch with most blood vessels has been proposed to localize the optic disc.

The retinal vasculature contains many vessels of varying length and thickness. In this method by means of determining the vessel network, the vessel with the maximum connections is selected. This branch is used to localize the optic disc. For this the binary blood vessel skeleton map is obtained.

For each skeleton pixel the amount of neighbor skeleton pixels is determined. If the amount of neighbors is smaller than three, then the pixel is added to the vessel-image. Otherwise the pixel is added to the branch-image. A vessel is a collection of points, starting at a point with only one neighbor and ending at a point with only one neighbor [15].The algorithm performs the following steps:

1. Select the branch vessel with most vessels connected to it. If there are a lot of such vessels, then the branch with the maximum number of branch pixels is selected.

2. Bounding box of the branch with the maximum vessel connections is taken.

3. The center of the bounding box is taken as the optic disc center (see Figure5).

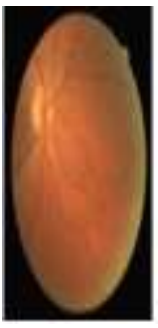

(a)

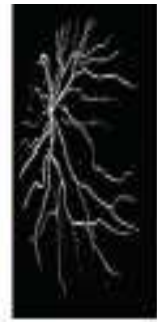

(b)

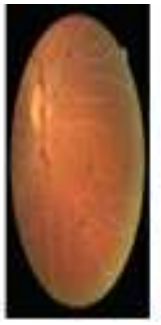

(c)

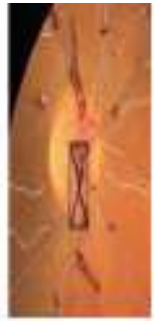

(d)

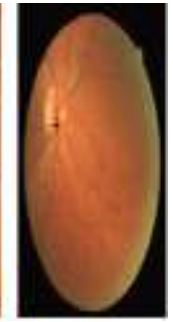

(e) 
Figure5. Figure5.Localization of Optic Disc (a) Original Color Retinal Image (b) The binary vessel map (c) Overlay of branch vessel network and the original image (d) The bounding box of the best branch and the determination of the optic disc center (e) Detected optic disc center ${ }^{15}$.

Since most of the times, the optic disc boundary is not well defined or it is hidden by blood vessels, a geometric contour model is used to detect the optic disc boundary in the retinal images. Firstly, the original image is I Lab space using color mathematical morphology. Then the blood vessels are removed by performing dilatation and erosion .Dilatation helps to remove the blood vessels and erosion restores the boundary to its original position. Geometric active contour model determines the boundary of the optic disc with variational formulation. In general, a snake is a set of points initially placed near the contour of interest, which are gradually brought closer to the exact shape of the desired region in the image. This is carried out through iterative minimization of an energy function comprising an internal and an external term [15].

\subsubsection{Exudate Localization}

1. Preprocessing-Convert RGB to HIS color space and apply local contrast enhancement for the distribution of the values of pixels around local mean to make further segmentation easy.

2. Image Segmentation-Image segmentation partitions image pixels on the basis of one or more selected image features e.g. color. Pixels with distinct color are separated into different regions (see figure6). Fuzzy C-means clustering allows pixels to belong to multiple classes with varying degree of membership [16].

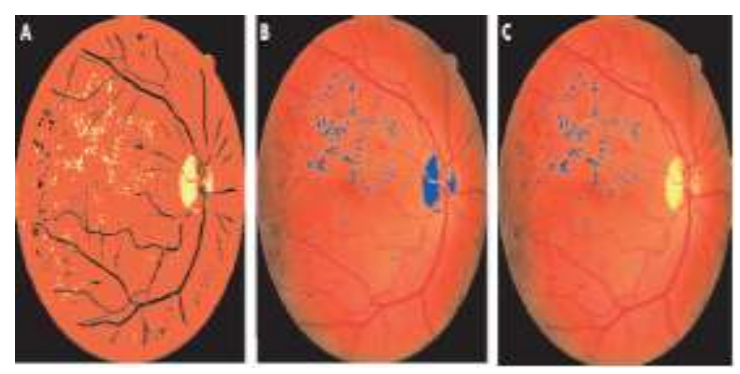

Figure 6. Figure6.Color image segmentation (A) FCM segmented image (B) candidate exudates region overlaid on the original image and (C) Final classification. ${ }^{16}$

3. Feature Extraction-In order to classify the segmented regions into exudate and non-exudate they must be represented with relevant and significant features to give them the best possible class seperability. False positive regions such as light reflections, cotton wool spots and most importantly optic disc should be sorted out. Optic disc localization can be automated by the methods described above. The segmented regions can be differentiated using features such as color, size, edge and texture.

4. Classification-Neural network can be used for classification with its input nodes corresponding to the feature set and the output node gives the final classification probability. In the object classification stage a three layer perceptron neural network with 18 input nodes corresponding to the feature set were used. Experimentation with a hidden layer with a range of 2-35 hidden units to find the optimum architecture (15 hidden units) was done. A single output node gave the final classification probability. FCM clustering was applied to the 75 color images of the prepared image dataset including 25 normal and 50 abnormal images. The outcome of the segmentation step was 3860 segmented regions consisting of 2366 exudates and 1494 non-exudates, which were considered negative cases. These regions were then labeled as exudates or non-exudates by a clinician to obtain a fully labeled dataset of examples. Two different learning methods, including standard back propagation (BP) and scaled conjugate gradient (SCG) descent were investigated for. The output threshold gives the balance between sensitivity and specificity. Sensitivity and specificity were adjusted by changing the threshold values.

\subsection{Detection of microaneurysms and hemorrhages}

\subsubsection{Vessel Extraction}

Retinal images have pathological noise and backgrounds with varied texture which lead to problems in vessel extraction .In order to remove the noise, gray opening operation and an opening by reconstruction with a linear structuring element are employed to the original image at various orientations (see Figure7). Secondly, the Top -Hat transform combined with reconstruction opening and closing operations are proposed to strengthen the smoothed image by iteratively filtering the image [17].

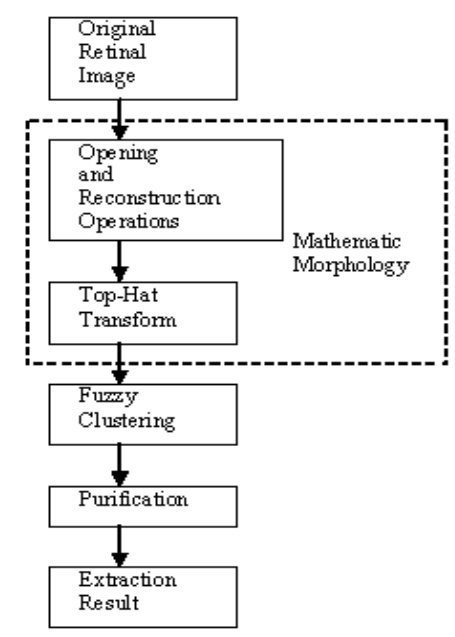

Figure 7. Flowchart of the proposed technique ${ }^{17}$

\section{Smooth image and remove noise}

The image is smoothed by gray mathematical morphology by selecting the structuring element. Here linear structuring elements based on the line type property of vessels have been used. However, it is important to note that an opening operation used by a linear structuring element will remove a vessel or some parts of it when the vessels in the image have orthogonal directions or the structuring element is longer than the vessel 
width. On the contrary, when the structuring element and the vessel have parallel directions, the vessel will never be changed [17]. Hence linear structuring element at different orientations has been used to get the maximal response. The length of the structuring element is close to the diameter of the largest vessel. Since the diameter of the largest vessels is approximately 6 pixels, the structuring element is taken to be 7 pixels long and 1 pixel in wide. Structuring elements are applied to perform a smoothening operation first by erosion and then dilatation

\section{Strengthening of vessels and removing background}

After the smoothening of the image the Top-Hat transform is applied to strengthen the vessels by selecting appropriate structuring elements .Here, the Top- Hat transform is applied to the smoothed image at 12 directions, and the computational results of the 12 directions are summed up to increase the gray difference between the vessels and the background[6]. The corresponding formula is as follows:

$S_{\text {sumx }}=\sum_{i=1}^{12}\left(S_{o p}-\gamma L_{t}\left(S_{\infty}\right)\right)$

However, most images containing noisy data need to be further smoothed by a Gaussian filter which has the width of 7 pixels, and further strengthen the curve feature of vessels by the use of Laplacian transform.

\section{Fuzzy clustering for vessel extraction}

After the retinal vessels in the image have been enhanced, the next step is to extract the vessels. Fuzzy clustering method is the most popular tool for to extract the vessel. Its an iterative clustering method that produces an optimal partition by minimizing the weighted-within group sum of squared errors. [17].When the partitioning is completed a defuzzification process takes place for the conversion of fuzzy partition to a crisp one. Finally a purification procedure is done and final extraction is achieved.

\subsubsection{Detection and Removal of Fovea}

The fovea is a dark region located in the centre of the macula. It appears in the diagnosis of hemorrhages and microaneurysms as optic disc in the automated detection of exudates. It is detected using the optic disc and the main blood vessel i.e. the thickest and the largest blood vessel arising from it. The course of the vessel can be obtained by seeing its continuity with the fovea and modeled as a parabola. The pixel on the main blood vessel which is closest to the centre of the optic disc is taken as the vertex of the parabola. The fovea is taken to be around 2-3 optical disc diameter distances from the vertex going along the main axis of the parabola that has been modeled and is considered to be the darkest region. Around 1 optic disc diameter around this center is taken to be the region of the fovea and that area is suppressed during detection of hemorrhages and microaneurysm.

\subsection{Retinal grading algorithm}

The distribution of exudates around the region of the fovea helps to determine the severity of the disease. The lesions close to the macular region are more dangerous than the once further away and require immediate intervention. In the same way the count, size and the distribution of the hemorrhages and microaneurysms help to determine the severity of the Retinopathy. The International Council of ophthalmology identifies five classes of Retinopathy of Diabetic origin such as none, mild moderate, severe and of proliferative state. The Retinal Grading Algorithm helps to automatically classify the DR (Diabetic Retinopathy) intensity based on these criteria [18].

\section{CONCLUSION}

The automated Diabetic Retinopathy diagnosis system is thus used to detect various lesions of the retina i.e. exudates, microaneurysms and hemorrhages and there count size and location to assess the severity of the disease so that the patient can be diagnosed early and referred to the specialist well in advance for further intervention. There are certain features present in the normal physiology of the retina which have to be differentiated from the abnormal pathology e.g. optic disc has the same pixel brightness as the exudates and thus has to be localized before establishing the presence of the exudates. Similarly the blood vessel and fovea region have to be subtracted from the retinal image before diagnosing microaneurysms and hemorrhages. Finally the Retinal Grading Algorithm, which follows the International council of Ophthalmology's criteria for the assessment of the severity of the disease, helps in establishing the severity of the disease so that the patient can accordingly be referred to the specialist and hence treated accordingly.

\section{REFERENCES}

[1] C. Sinthanayothin, J. F. Boyce, H. L. Cook, and T. H.Williamson, "Automated localization of the optic disc, fovea and retinal blood vessels from digital color fundus images," $\mathrm{Br}$. J. Opthalmol., vol. 83, pp. 231-238, Aug. 1999.

[2] S. Tamura and Y. Okamoto, "Zero-crossing interval correction in tracing eye-fundus blood vessels," Pattern Recogn., vol. 21, no. 3, pp. 227-233, 1988.

[3] A. Pinz, M. Prantl, and P. Datlinger, "Mapping the human retina,” IEEE Trans. Med. Imag., vol. 1, pp. 210-215, Jan. 1998.

[4] K. Akita and H. Kuga, "A computer method of understanding ocular fundus images," Pattern Recogn., vol. 15, no. 6, pp. 431443, 1982.

[5] F. Mendels, C. Heneghan, and J.-P. Thiran, "Identification of the optic disk boundary in retinal images using active contours," in Proc. Irish Machine Vision Image Processing Conf. (IMVIP'99), Sept. 1999, pp. 103-115.

[6] João V. B. Soares, Jorge J. G. Leandro, Roberto M. Cesar Jr., Herbert F. Jelinek, and Michael J. Cree, Retinal Vessel Segmentation Using the 2-D Gabor Wavelet and Supervised 
Classification IEEE TRANSACTIONS ON MEDICAL IMAGING, VOL. 25, NO. 9, SEPTEMBER 2006

[7] Frédéric Zana and Jean-Claude Klein, Segmentation of Vessel-Like Patterns Using Mathematical Morphology and Curvature Evaluation ,IEEE TRANSACTIONS ON IMAGE PROCESSING, VOL. 10, NO.7, JULY 2001

[8] Subhasis Chaudhuri, Shankar Chatterjee, Norman Katz. Mark Nelson and Michael Goldbaum, Detection of Blood Vessels in Retinal Images Using Two-Dimensional Matched Filters, IEEE TRANSACTIONS ON MEDICAL IMAGING, VOL. 8, NO. 3, SEPTEMBER 1989

[9] Tamar Peli and Eli Peli, Fundus Image Analysis Using Mathematical Morphology, in Vision Science and Its Applications, 1994 Technical Digest Series, Vol. 2 (Optical Society of America, Washington, DC, 1994), pp. 224-227.

[10] Thomas Walter and Jean-Claude Klein, Segmentation of Color Fundus Images of the Human Retina: Detection of the Optic Disc and the Vascular Tree Using Morphological Techniques, J. Crespo, V. Maojo, and F. Martin (Eds.): ISMDA 2001, LNCS 2199, pp. 282-287, 2001. @ Springer-Verlag Berlin Heidelberg 2001

[11] Carla Agurto, Victor Murray, Eduardo Barriga, Sergio Murillo, Marios Pattichis, Herbert Davis, Stephen Russell, Michael Abràmoff, and Peter Soliz, Multiscale AM-FM Methods for Diabetic Retinopathy Lesion Detection, IEEE Trans Med Imaging.2010 February; 29(2):502-512
[12] D.Abraham Chandy, V.Vijaya Kumari. Genetic Algorithm Based Location of Optic Disc in Retinal Images.Academic Open Internet Journal Volume 17, 2006.

[13] H. Li, O. Chutatape. "Automatic Location of Optic Disc in Retinal images”. IEEE ICIP, 2001, pp. 837-840.

[14]H. Li and O. Chutatape. "Fundus image features extraction". Proceedings of the $22^{\text {nd }}$ Annual International Conference of the IEEE Engineering in Medicine and Biology Society, Vol. 4, 2000, pp.3071 -3073.

[15] Giri Babu Kande, T. Satya Savithri, P. Venkata Subbaiah, M. R. N. Tagore. Automatic detection and boundary estimation of optic disk in fundus images using geometric active contours. J Biomedical Science and Engineering, 2009, 2, 90-95.

[16] A Osareh, M Mirmehdi, B Thomas, R Markham. Automated identification of diabetic retinal exudates in digital colour images. Br J Ophthalmol 2003; 87:1220-1223.

[17] Yong Yang, Shuying Huang, Nini Rao. An Automatic Hybrid method for Retinal blood vessel Extraction. Int. J. Appl. Math. Comput. Sci., 2008, Vol. 18, No. 3, 399-407.

[18] Automated Feature Extraction for Early Detection of Diabetic Retinopathy in Fundus Images. www.cse.iitm.ac.in. 